\title{
On-Orbit Measurement of TOPEX/Poseidon Altimeter Antenna Pattern
}

$$
\begin{gathered}
\text { Nasia } 11 \\
12-19 \\
5=+5 \\
9010
\end{gathered}
$$

\author{
PHILIP S. CALLAHAN \\ DAVID HAUB
}

Jet Propulsion Laboratory

California Institute of Technology

Pasadena, California, USA

\begin{abstract}
The NASA and CNES altimeters on the TOPEXIPoseidon satellite share a $1.5-\mathrm{m}$ antenna. Early data from the NASA altimeter suggested that the beam was broader than measured preffight. An altimeter transponder was modified to output a relative measurement of received power. The instrument is briefly described. The instrument was deployed on the TOPEX ground track at the coast near Los Angeles. California. Measurements from three of these deplovments are presented to show the on-orbit antenna pattern. The measured pattern is effectively broader than preflight in the central region. particularly the part of the patsern which corresponds to the tail of the altimeter waveform where the attitude is determined. This result is consistent with the general shape of both the TOPEX and Poseidon waveforms. As the TOPEX corrections which depend on values from the tail of the waveform have been compensated for deviations from the preflight measurements, no appreciable effect on the final data is expected.
\end{abstract}

Keywords Altimeter, antenna pattern, TOPEX. Poseidon

Satellite altimeters make extremely precise and accurate measurements of the range to the sea surface. In order to achieve an altimeter's ultimate accuracy, many effects must be accounted for. One important effect is error in the range caused by differences between the waveform observed by the altimeter and the simplified model waveform used to design the range tracking algorithms. The difference between the observations and the model is mainly a function of satellite attitude and significant waveheight (SWH). SWH is measured by the altimeter as part of the range tracking process. The apparent satellite attitude can be determined from the "tail" of the waveform (see Brown, 1977; Rodriguez, 1988; Chelton et al., 1989). Unfortunately, the TOPEX waveforms have a number of instrumental features which complicate this determination (Hayne et al., 1994). In addition, during early attempts to determine the satellite attitude from waveform data it appeared

This research was carried out by the Jet Propulsion Laboratory, California Institute of Technology under contract with National Aeronautics and Space Administration. We would like to thank D. G. Kubitschek of the University of Colorado for carrying out the yaw computation for the onorbit measurements.

Author correspondence to Dr. Philip S. Callahan. Mail Stop 30(0)-3/9. Jet Propulsion Laboratory, Pasadena, California 91109-8099. USA. email: psecre purim jpl nasa gov 
that there was more energy than expected in the tail. While this could be part of the other waveform features, it could also arise from the altimeter antenna pattern being broader than was measured on the ground preflight.

From other verification activities planned for TOPEX/Poseidon (Christensen and Menard, 1992), an altimeter transponder was available that could be modified to make measurements of the on-orbit antenna pattern. The instrumentation, data acquisition and reduction, and results of those measurements are presented here. We find that the onorbit antenna pattern has a 5-15\% enhancement between $0.2^{\circ}$ and $0.7^{\circ}$ from the center compared to the preflight ground measurements. These differences are comparable to other waveform features described by Hayne et al. (1994).

\section{Experimental Setup}

\section{Instrumentation}

The altimeter transponder was modified by disabling the transmitter section and running the receiver output to power measuring circuitry as shown in Figure 1 . A $35-\mathrm{cm}$ dish antenna collects the signal which is immediately amplified at the feed in order to reduce system noise. After passing through the transponder amplifiers, a tunnel diode detector

\section{Receiver to Record Topex Antenna Pattern}

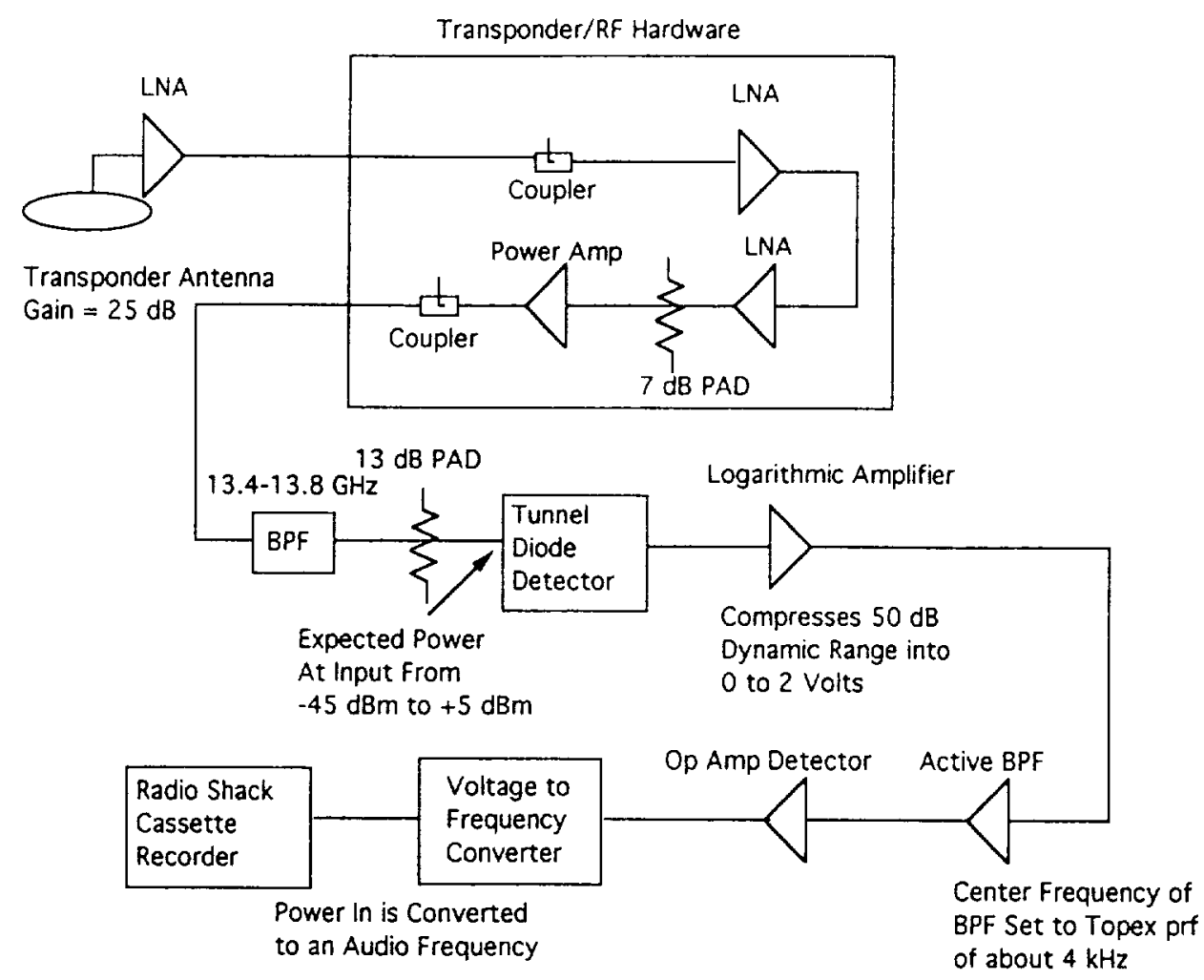

Figure 1. Measurement system block diagram. 
and logarithmic amplifier convert the RF input to a 0 - to $2-\mathrm{V}$ signal at the pulse repetition frequency (PRF). An active bandpass filter and integrator produce a voltage proportional to the received power. This output drives a voltage-to-frequency converter operating in the audio range. The resulting tone is recorded on an analog tape recorder. It should be noted that the transponder antenna beam width (approximately $5^{\circ}$ ) does not affect the measurements since we are in the far field of the TOPEX antenna. Also, the gain of the transponder antenna is important only to the extent of determining the measurement system SNR.

The measurement system was redesigned after several of the early measurements (not reported here) in order to be less sensitive to the exact altimeter PRF. The system was slightly temperature-sensitive, but this was accounted for both by temperature stabilization at $45^{\circ} \mathrm{C}$ and by calibration. The system was calibrated over a range of $25 \mathrm{~dB}$ of input at $45^{\circ} \mathrm{C}$ and room temperature (approximately $25^{\circ} \mathrm{C}$ ) in the laboratory. The calibration was repeated twice during December and three times in January, and showed no significant changes. These calibration curves are shown in Figure 2. The curves separate the most at the highest power levels/frequencies. The maximum observed frequency was about $5200 \mathrm{~Hz}$ and the minimum used in the analysis was $120 \mathrm{~Hz}$. While the curves differ by up to $0.6 \mathrm{~dB}$, even with temperature calibration considered, the difference between the extreme calibration curves gives a difference of about $3 \%$ in the retrieved relative power used here for frequencies between about 3 and $5.2 \mathrm{kHz}$. Calibration-induced differences are less at lower frequencies (lower relative power). Typical observation fluctuations of $50 \mathrm{~Hz}$ at $5 \mathrm{kHz}$ and $10-20 \mathrm{~Hz}$ at low frequencies correspond to less than $2 \%$ variations in the final pattern.

Calculations indicated that the system would have an 18-dB signal-to-noise ratio. The observations showed a sidelobe at $-16 \mathrm{~dB}$ basically confirming the calculations. The observations show a noise floor of about $1-2 \%$ (see Figure $5-7$ ). Overall we believe that the measurement system was able to measure the relative power received from TOPEX over a range of about $16 \mathrm{~dB}$ to $3-4 \%$ at worst and $1-2 \%$ if appropriate calibration curves are available.

\section{Data Collection}

TOPEX/Poseidon is in a 10-day exact repeat orbit which is maintained within $\pm 1 \mathrm{~km}$. Pass 119 strikes the southern California coast near San Pedro (Los Angeles). We located a suitable site about $1 \mathrm{~km}$ east of the groundtrack on a bluff approximately $30 \mathrm{~m}$ above the ocean (latitude $=33^{\circ} 42^{\prime}$, east longitude $=241^{\circ} 22^{\prime}$ ). The altimeter was in lock over the water as it approached the coast but lost lock over land. We do not believe that the change in signal modulation affected our measurements.

Observations were made on every repeat cycle from November 6, 1992 to February 3, 1993 (except Christmas). Various problems with the early measurements (PRF sensitivity and inadequate calibration) and some later measurements (a failing temperature controller introduced audiofrequency noise and also sent the system outside of the calibrated range) resulted in the three apparently complete and reliable cuts through the antenna pattern reported here.

\section{Data Reduction}

The audio tone representing the received power was extracted from the tape using an analog-to-digital converter with MathLab software running on a Macintosh computer. A 


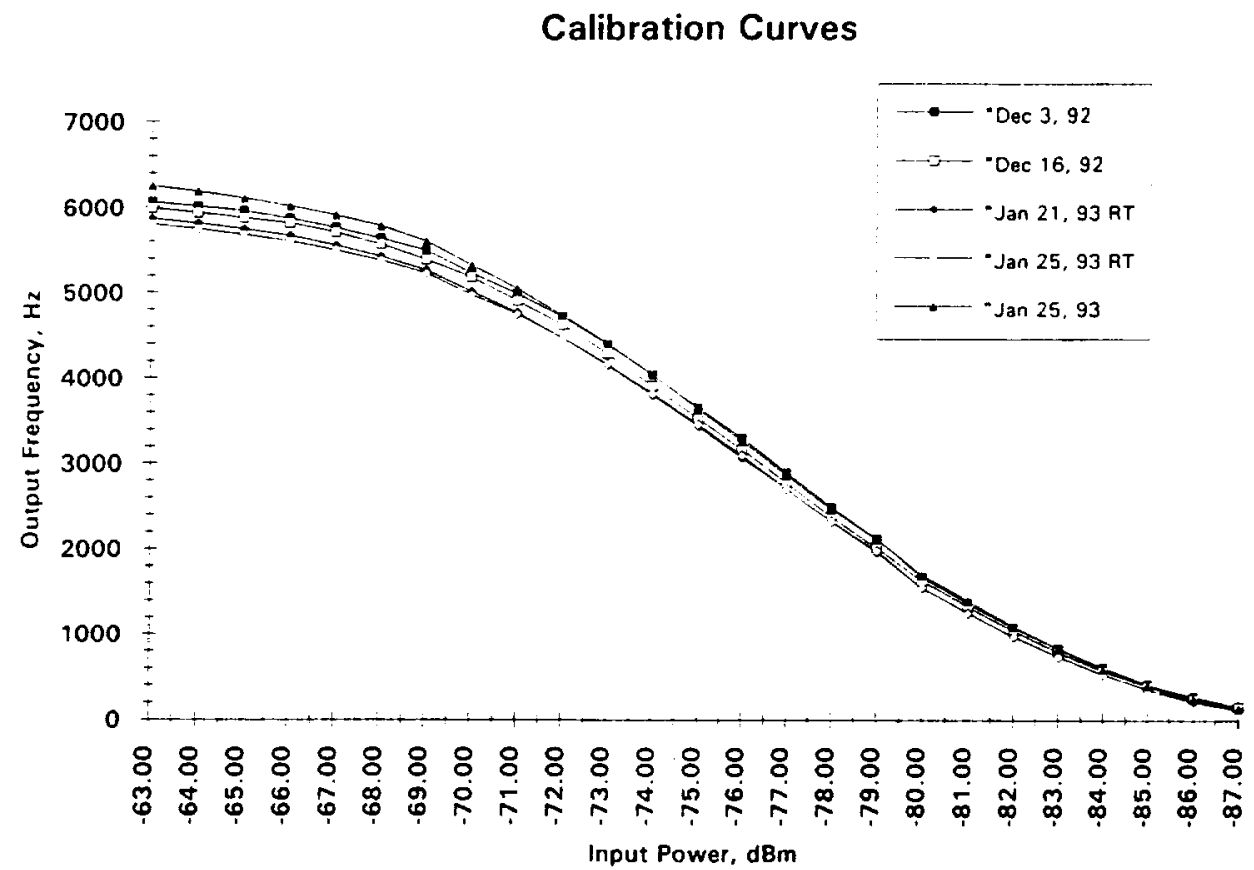

Figure 2. Calibration curves from December 1992 and January 1993 showing variations with time and temperature. RT indicates room temperature calibration. The other calibrations were done with heaters maintaining the electronics at $45^{\circ} \mathrm{C}$.

plot of the frequency vs. time output for January 24, 1993 is shown in Figure 3. As can be seen in the figure, the output has rapid variations at high received frequencies. This occurs because the MathLab digitizing interval depends on input frequency. The resulting frequency vs. time data were converted to power using the calibration curves described above and to angle using satellite orbit geometry. All observations have been normalized to unity at the maximum and interpolated to equal angular intervals $\left(0.05^{\circ}\right)$. The discussion below is carried out in percent of the maximum ("power," not $\mathrm{dB}$ ).

The preflight ground antenna measurements made by the Johns Hopkins Applied Physics Laboratory were supplied as strip chart output by C. Purdy of Wallops Flight Facility, Goddard Space Flight Center. Four cuts were made through the antenna pattern. These measurements were hand-digitized at an angular interval of $0.05^{\circ}$ and are shown in Figure 4. The strip charts appear to have noise of about $0.2 \mathrm{~dB}(5 \%)$.

The orientation of the antenna relative to the satellite is known, and the satellite attitude (yaw) during the on-orbit measurements has been reconstructed (Kubitschek, private communication). The preflight measurements were linearly interpolated to the cuts observed in the on-orbit measurements.

\section{Results}

The measurements from December 5, 1992, January 14, 1993, and January 24, 1993 are shown in Figures 5-7. Each figure shows the on-orbit measurement, the interpolation of the preflight measurements, and the difference between them. The full patterns as well 


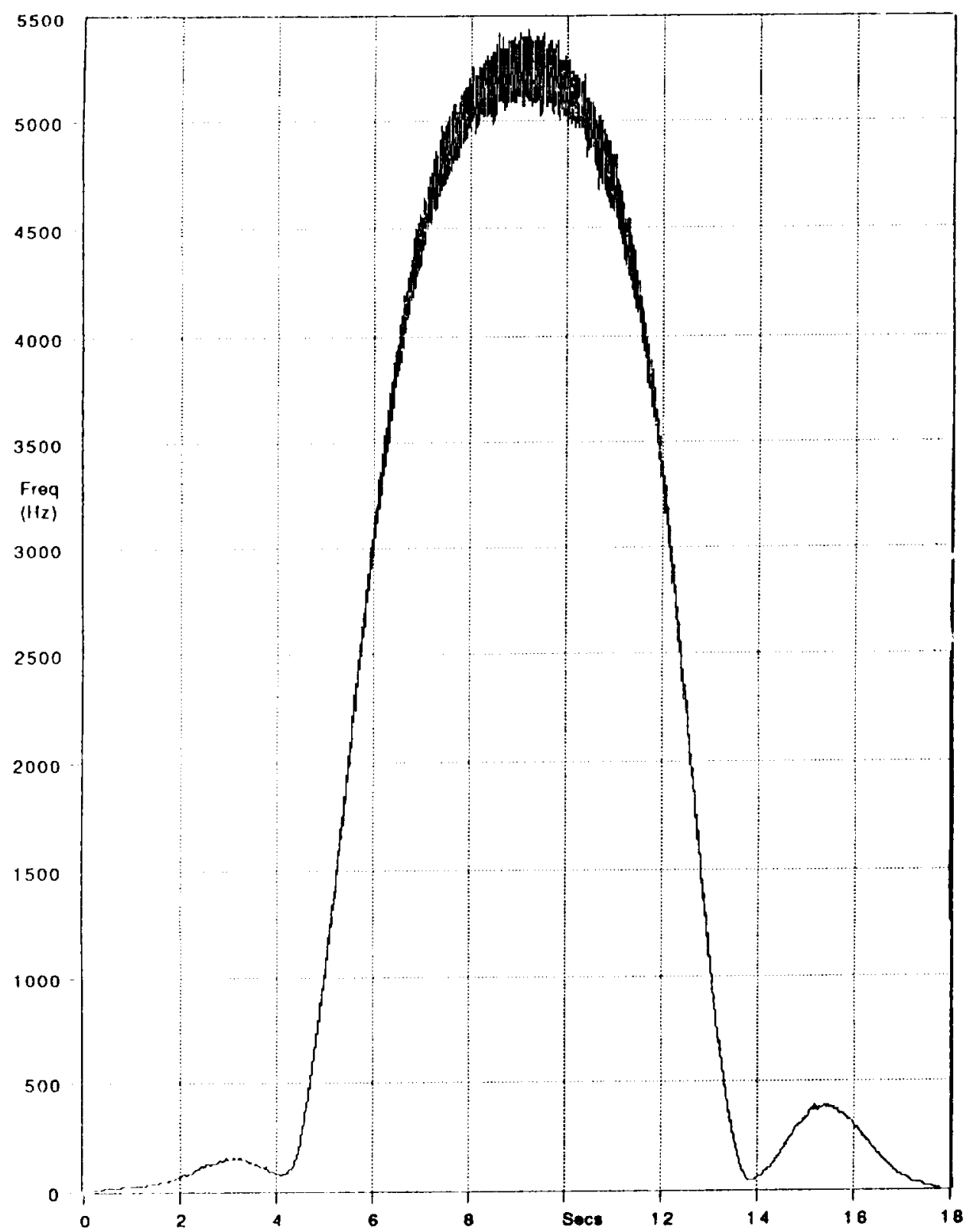

Figure 3. Frequency vs. time as retrieved from measurement tape for observation of January 24 , 1993.

as blow-ups of the peak and residual are shown. Figures 5-7 show that the on-orbit patterns are broader or "squarer" around the peak than the preflight pattern. This results in differences of $5-15 \%$ in the regions from 0.2 to $0.7^{\circ}$. The difference is approximately $10 \%$ near $0.45^{\circ}$ where the attitude determination gates fall. The position of the on-orbit nulls is similar (approximately $\pm 1.2^{\circ}$ ) to the preflight values. Except for December 5, 

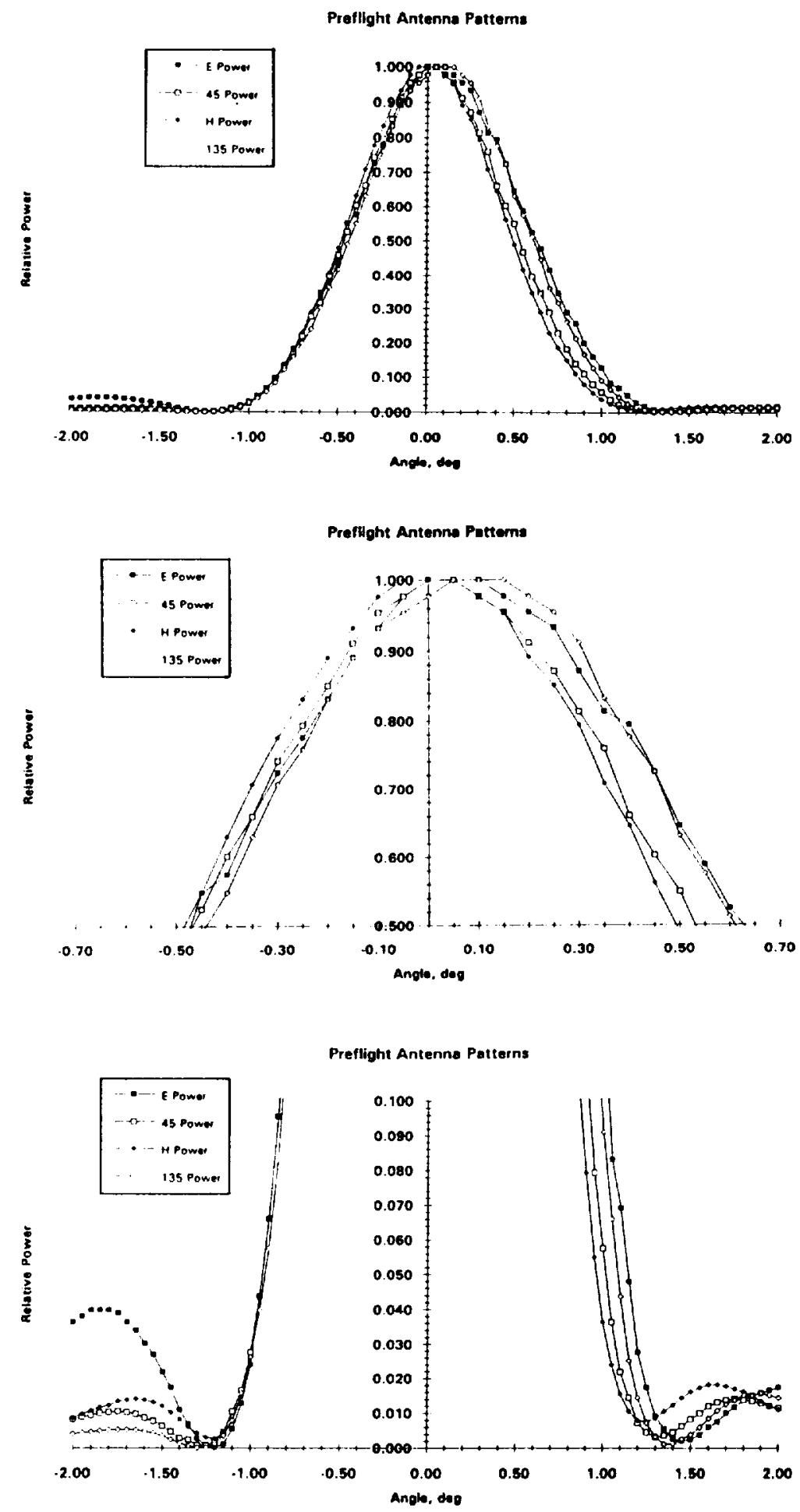

Figure 4. Four cuts of the antenna pattern as measured on the ground. E cut, solid square; $45^{\circ} \mathrm{cut}$, open square; $\mathrm{H}$ cut, solid diamond: $135^{\circ}$ cut, open diamond. 

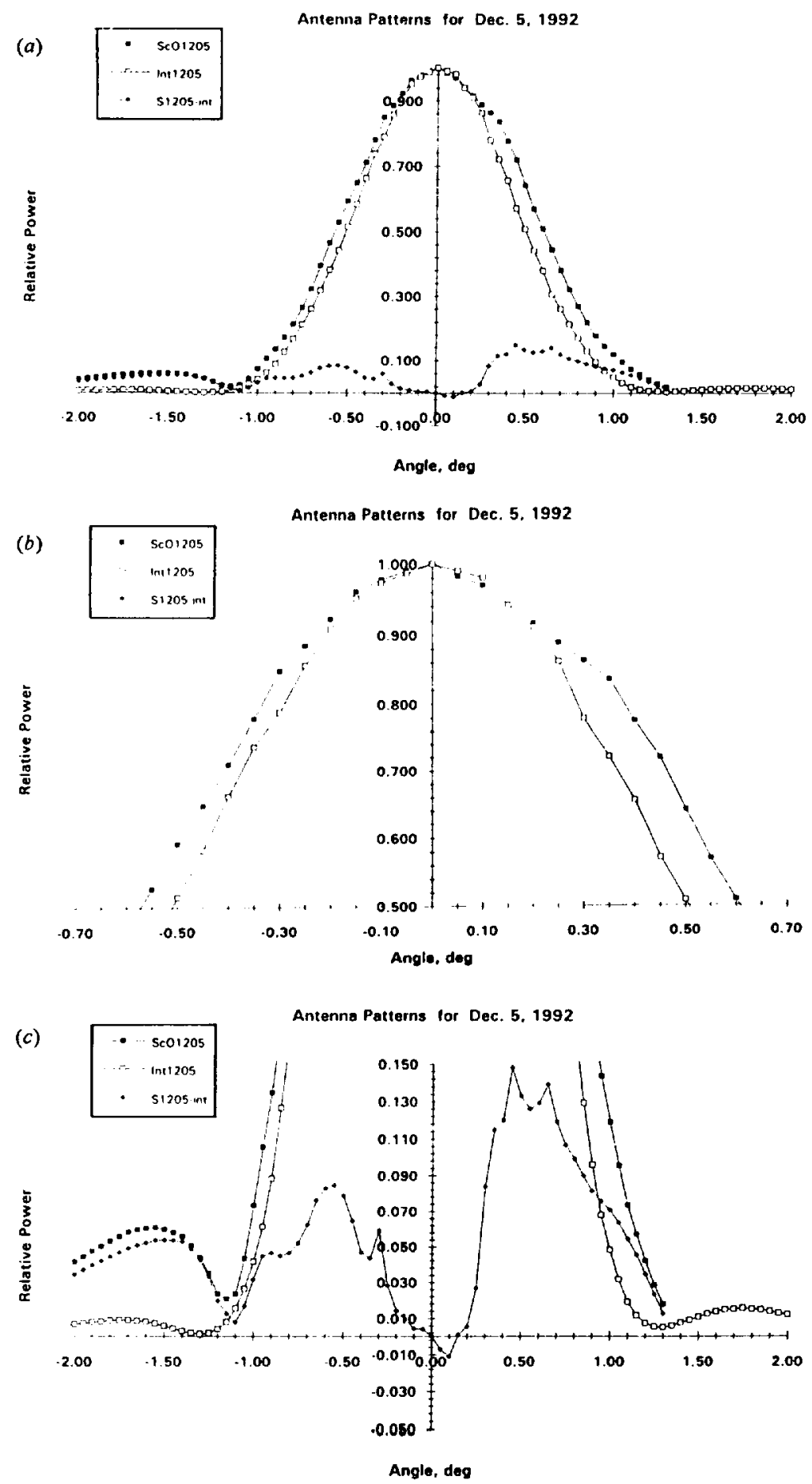

Figure 5. Measurements for December 5, 1992. On-orbit antenna pattern measurements compared to the interpolated ground measurement. (a) Overall pattern. (b) Center to half power point. (c) Lowest $10 \%$. On-orbit measurement, solid square; interpolated prelaunch pattern, open square; difference, orbit-interpolated, solid diamond. 


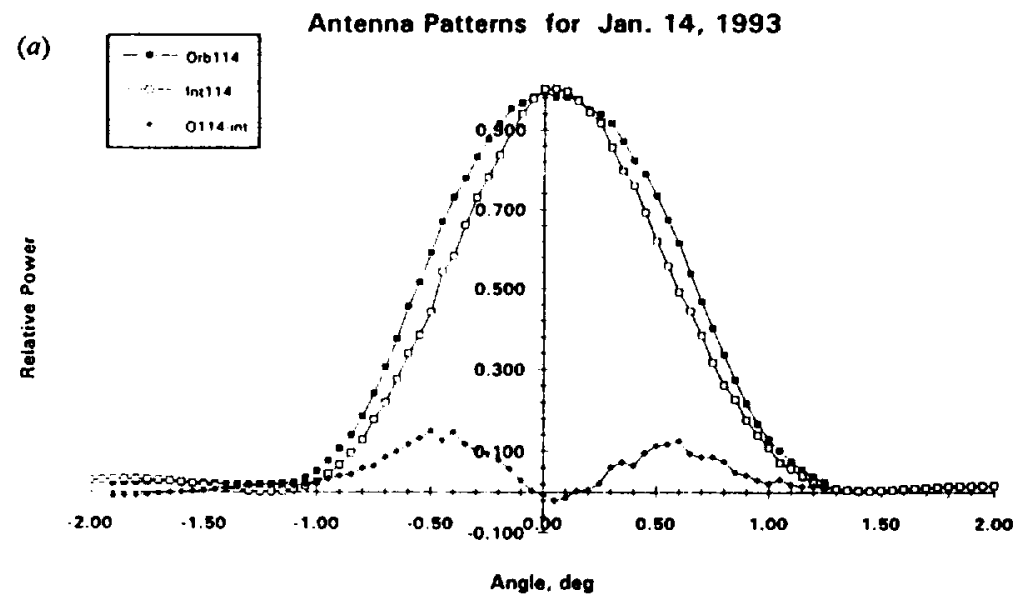

(b)

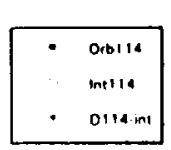

Antenna Patterns for Jan. 14, 1993

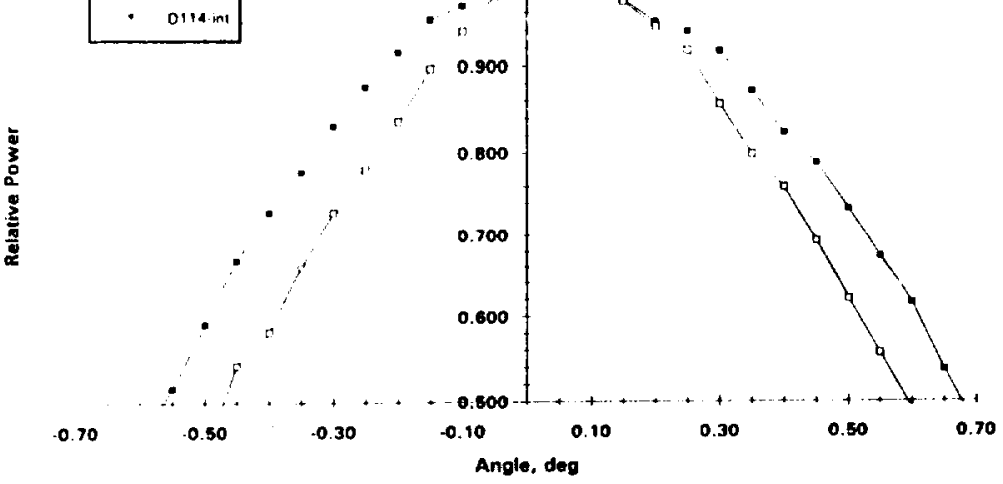

(c)

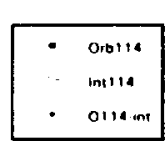

Antenna Patterns for Jan. 14, 1993

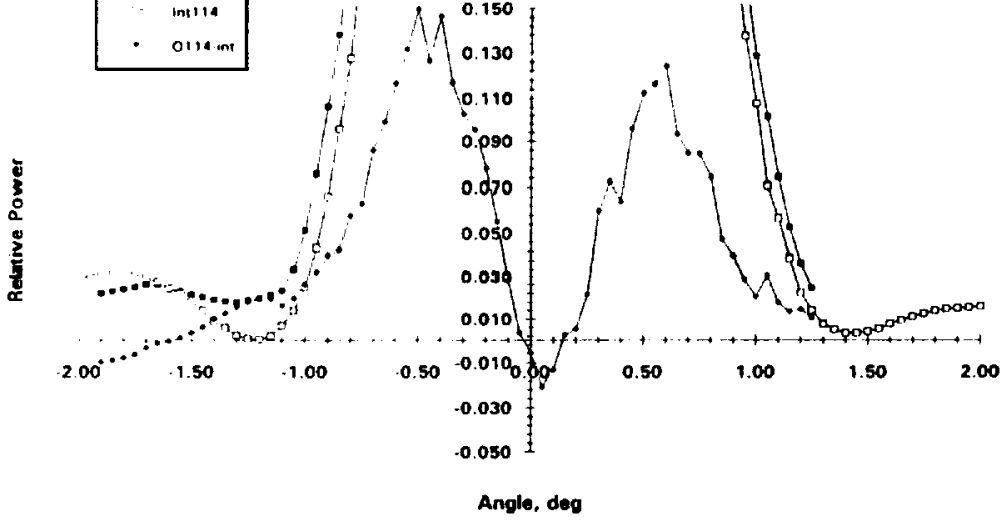

Figure 6. Measurements for January 14, 1993. On-orbit antenna pattern measurements compared to the interpolated ground measurement. (a) Overall pattern. (b) Center to half power point. (c) Lowest $10 \%$. On-orbit measurement, solid square; interpolated prelaunch pattern, open square; difference, orbit-interpolated, solid diamond. 


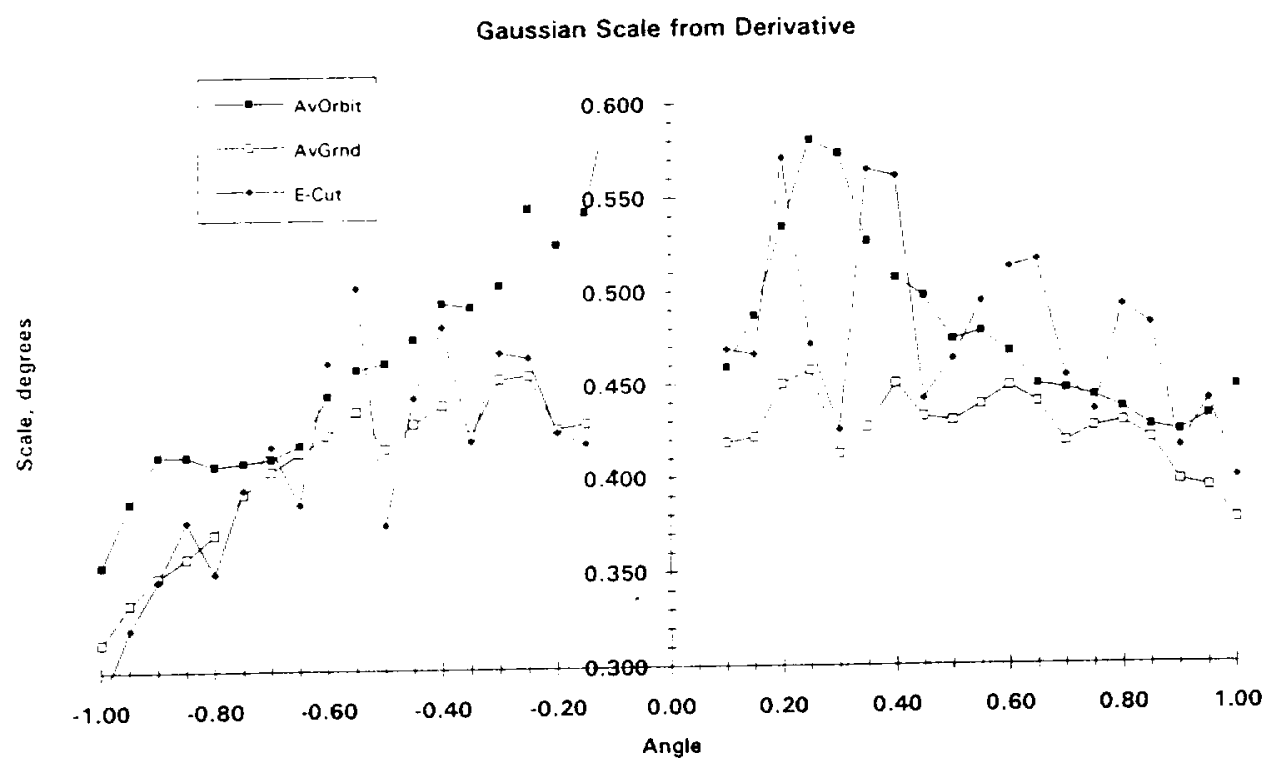

Figure 8. The scale of a Gaussian, $\theta_{\ldots}$, from the numerical derivative of the pattern for the average on orbit (solid square), average preflight (open square), and the preflight E cut (solid diamond) patterns.

noise in the derivative. This method provides numerically reliable values between about $0.1^{\circ}$ and $1.0^{\circ}$. The scale is fairly constant for the average of the ground measurements at 0.42 . The E-cut and on-orbit data are somewhat noisier and give wider values: about 0.46 for the E-cut and 0.47 for the on-orbit data. As seen in the other analysis, the on-orbit data are better represented by a broader function than the ground measurements in the central region.

\section{Discussion and Conclusions}

The excess antenna gain in the $0.7-0.2^{\circ}$ area will result in excess waveform power in gates $54-128$ ( 128 is the maximum, i.e., the altimeter only uses the center of the antenna beam). which will appear in telemetry waveform samples 43-64 (Hayne et al., 1994). Angles between $\pm 0.17^{\circ}$ are used in the altimeter AGC determination which sets the overall scale for the waveform. Angles around $0.45^{\circ}$ affect the waveform signal used for attitude determination and for pointing angle/sea state corrections to other measured quantities. The residuals here show typical differences of about $5-7 \%$ between the relative power in the $0-0.2^{\circ}$ region and the $0.45^{\circ}$ region. This is similar to other features reported by Hayne et al. (1994). CNES altimeter (Poseidon) data are also consistent with the results reported here (O.-Z. Zanife, private communication).

For processing of TOPEX waveform data the important parameter is the Gaussian antenna width used. Data from Table 1 give a value for $\theta_{01}$ in a Gaussian of 0.47 (full width at half maximum of $1.11^{\circ}$ ) from the on-orbit measurements. A similar value is deduced from the numerical derivative in Figure 8 . The value originally used by Hayne and Rodriguez (private communication) in computing waveform effects was 0.44 (FWHM $=1.04^{\circ}$ ). Later, the value used by them was increased to 0.46 based on early results 
from these measurements. This difference could change polynomial corrections for pointing angle/sea state effects depending on how the waveform correction factors are computed. Waveform corrections now largely absorb the excess power from the antenna factor into that for other features. Thus, the attitude angle and pointing angle/sea state corrections for TOPEX data produced on the Geophysical Data Records do not suffer from the apparent broadening of the antenna pattern. In future altimeters, on-orbit measurement of the antenna pattern should be carried out to provide confidence in quantities based on the altimeter waveform.

\section{References}

Brown, G. S. 1977. The average impulse response of a rough surface and its applications. IEEE Trans. Antennas Propagation AP-25:67-74.

Callahan, P. S. 1992. TOPEX GDR Users Handbook. JPL Internal Document, JPL D-8944.

Chelton, D. B., E. J. Walsh, and J. L. MacArthur. 1989. Pulse compression and sea level tracking in satellite altimetry. J. Atmos. Oceanic Tech. 6:407-438.

Christensen, E. J., and Y. Menard, eds. 1992. TOPEX/Poseidon joint verification plan. JPL Publication 92-9, available from JPL Document Review, Jet Propulsion Laboratory, Pasadena, Calif.

Hayne, G., D. W. Hancock III, C. Purdy, and P. S. Callahan. 1994. The corrections for significant wave height and attitude in the TOPEX radar altimeter. Journal of Geophysical Research 99:24491-24955.

Rodriguez, E. 1988. Altimetry for non-Gaussian oceans: Height biases and estimation of parameters. Journal of Geophysical Research 93:14,107-14,120. 